\title{
CON EL DOS ¿NACIÓ LA PENA? EL SURGIMIENTO DE LA SUBJETIVIDAD EN LA ALTERIDAD DESDE LA OBRA DE EMMANUEL LEVINAS
}

\author{
WITH THE TWO ¿BORN THE PENALTY? THE ORIGIN OF \\ SUBJECTING IN ALTERITY FROM EMMANUEL LEVINAS' WORK
}

Diego Fonti

\begin{abstract}
RESUMEN
Este trabajo parte de exponer el "acabamiento" - tanto en el sentido de terminación como en el de plena realización - del sujeto moderno, que se creyó capaz de estructurar, legalizar y manipular la realidad toda por el propio poder de su conciencia. Para ello se indagará cómo opera en la obra de Husserl la conciencia intencionalmente estructurada, y el juicio que Heidegger formula sobre esta comprensión y su propia fenomenología de la existencia. A partir de la esta presentación, se indagará cómo Levinas sigue y supera a Heidegger, afirmando que éste es incapaz de dar cuenta del "origen" de la subjetividad humana: la nominación e in-vocación del Otro. El momento central de este artículo analiza el surgimiento de la subjetividad a partir de la respuesta a esta invocación.
\end{abstract}

PALABRAS CLAVE

Sujeto, Subjetividad, Fenomenología, Alteridad

\begin{abstract}
This paper begins with the "ending" - both in regards to its termination as well as of its full completion - of the modern subject, which was believed able to structure, legalize and manipulate the whole of reality by the very power of its consciousness. In this regards it will be analyzed how consciousness, intentionally structured, operates in Husser's work, and the Heidegger's judgment of this understanding, as well as his own phenomenology of existence. Starting with this presentation, it will be researched how Levinas follows and goes beyond Heidegger, by affirming that he is unable to account for the "origin" of human subjectivity: the nomination and invocation of the Other. The main moment of this article analyses the birth of subjectivity in the answer to this invocation.
\end{abstract}

\section{KEY WORDS}

Subjec, Subjectivity, Fenomenology, Alterit

Universidad Católica de Córdova, CONICET: diegofonti@gmail.com 
... que yo no sería, si tú no fueses en mí.

Con un bosquejo cuasi caricaturesco - no en sentido peyorativo, sino como fidelidad al original en forma de clave mínima de interpretación de una lectura en sí misma inabarcable, pero accesible gracias a esa llave - de las denominadas ciencias humanas en general y de la filosofía en particular, se podría postular una sucesión epocal en cuatro viñetas. En primer lugar se halla en el mundo griego una copertenencia entre la "realidad" y el "sujeto" pensante (ambas denominaciones son anacrónicas), copertenencia justificada por una norma o logos común, una estructura del mundo inteligible y accesible a un ser inteligente. El orden de la realidad y el orden del pensar estarían así fundados sobre una lógica previa común, un orden al cual habría de apegarse quien quisiera entender al mundo y entenderse. Esta estructura permanece incólume en el medioevo, donde el ingreso de un nuevo actor, el Dios monoteísta, considerado origen, orden y fin del mundo y del ser humano, no altera lo esencial de la noción griega del orden, cosmos, pero fundamenta toda multiplicidad en ese origen personal. Por supuesto que hay excepciones a este esquema, pero lo central es plantear que recién desde la modernidad se rompe la idea de una estructura común e inteligible para quien supiera "leer" la realidad, y que desde entonces es el sujeto pensante qua conciencia quien se ve a sí mismo como fundamento del orden, del conocimiento y del manejo del mundo.

Si el mundo griego puede leerse como un gran lamento por la unidad perdida del uno primordial - sin cuya ley hay hybris y culpa -, lo que la tradición pitagórica identifica con la pena nacida al ser ya dos, o sea, por la aparición del Otro - la multiplicidad - ante el sujeto, la modernidad pretende conjurar esa pena y su riesgo inherente con un "giro copernicano": ya no es un mundo a contemplar el que revelará una ley, nomos, inherente, sino que el sujeto consciente en su ratio será quien objetive, explique y ordene al mundo. Prometeo es la figura de ese sujeto, que significa una crítica de la dependencia de todo tipo de alteridad normativa, y por lo tanto se identifica a sí mismo como divinidad (Hinkelammert, 2005:11). Aunque Descartes, el punto de partida de esta modernidad, sigue la tradición agustiniana no sólo en las metodologías de autoindagación y duda sistemática omniabarcante, sino también en su hallazgo de una alteridad insita al sujeto que no ha sido gestada por el sujeto - la idea de infinito -, sin embargo la historia de los efectos de su planteo es la de un encierro de la conciencia en sí, un repliegue sobre la única certeza posible, la existencia del propio pensamiento, y la reconstrucción de un mundo objetivado sólo a partir de éste.

Luego de estos tres momentos abarcativos, o para citar a F. Rosenzweig (1997:43), luego de esta triple reducción sucesiva de la multiplicidad a una totalidad bajo uno de sus elementos irreductibles (mundo, Dios, ser humano), aparece un "fenómeno" que rompe con las certidumbres hasta entonces logradas. Siguiendo al pensador judeoalemán, el círculo identitario elaborado de Jonia a Jena, desde los primeros filósofos naturalistas hasta la identificación de ser y pensar en Hegel y el desbaratamiento de su sistema en la $1^{\text {a }}$ Guerra Mundial, se muestra imposible de cerrar, o desde siempre ya irreparablemente desfasado por una comunicación fundamental con Otro. Con palabras de Levinas, el periplo de Ulises, que abarca al mundo en su totalidad y vuelve a la tierra natal destruyendo toda diferencia, se demuestra no sólo falaz, 
sino simplemente imposible. Se trata del "descubrimiento" contemporáneo: la insuperable alteridad. La alteridad es insuperable en el sentido técnico hegeliano de "superar", que entiende que la conciencia - toda conciencia como parte de una conciencia absoluta - realiza un proceso histórico de enajenamiento, negación, recuperación y superación, por el cual toma conciencia de sí y se hace saber absoluto, saber del saber, noesis noeseos. Por el contrario, contra ese devenir "sin resto" el Otro se manifiesta inconceptualizable, superando toda donación de sentido abarcativa por parte de la propia conciencia.

En lo que sigue se presentará en primer lugar, de modo propedéutico, el "acabamiento" de ese sujeto moderno, que se cree capaz de estructurar, legalizar y manipular la realidad toda por el propio poder de su conciencia. Se lo hará entendiendo "acabamiento" tanto en el sentido de fin como en el sentido de plena realización. Para ello se indagará cómo opera en la obra de Husserl la conciencia, intencionalmente estructurada, ante la aparición del otro. El resultado de esta filosofía husserliana de la conciencia es puesto en juicio por Heidegger, que ve al ser humano como un desenvolvimiento práxico vital del propio ser, en el cual el "ser humano" no es sujeto sino Dasein, un "estar ahí" - no en el sentido local, sino como estar despierto ante la propia existencia - cuya primordial actividad es la cura, el cuidado por el ser y sus propias posibilidades. Levinas seguirá a Heidegger en su crítica a Husserl, pero verá que este centrarse en el cuidado por el ser, es incapaz de dar cuenta del "origen" de la subjetividad humana: la nominación e invocación del Otro (Casper, 2008:257). El análisis del surgimiento de la subjetividad a partir de la respuesta a esta in-vocación será el momento central del presente artículo.

\section{Acabamientos del sujeto: Husserl y Heidegger}

\subsection{La intersubjetividad monadológica de Husserl}

El método fenomenológico expuesto por Husserl tiene por leitmotiv "ir a las cosas mismas", e.d. centrar el análisis filosófico en aquello que se manifiesta a la conciencia en su modo de aparición, incluida la conciencia misma. Hacer fenomenología significa dejar los debates de escuelas y sus argumentos, para enfocarse en la aparición de lo dado a la conciencia y su descripción. Al hacerlo, la conciencia revela su estructura fundamental: toda conciencia es conciencia de algo. Esto es denominado intencionalidad de la conciencia, su estar dirigida a algo (Husserl, 1992a:83). No hay tal cosa como una conciencia vacía, que se irá llenando de contenidos o irá desplegando actividades, sino que la conciencia es esa correlación, y la fenomenología su investigación.

Para exponer esa correlación, se precisa abandonar la comprensión o disposición natural ante el mundo (Husserl, 1992b). Este abandono es denominado epoché o reducción, puesta entre paréntesis de toda creencia o juicio sobre lo trascendente a la conciencia para ganar su inmanencia. De este modo se priva de dar juicios sobre el mundo exterior, dejando de lado las creencias naturales sobre él, fundamentalmente la de su existencia trascendente a la propia conciencia.

Intentio e intentum son inseparables, esto es, todo acto de la conciencia se muestra como correlación de acto y objeto, pero ambos suceden en el marco de la conciencia subjetiva. El "objeto", noema, indica el cómo de sus determinaciones para la conciencia, el modo en que la 
conciencia se relaciona con sus objetos, mientras que el "acto" que dona un sentido a las determinaciones que aparecen es denominado noesis (Husserl 1992a:205). A la luz de ese doble componente del fenómeno en su manifestación inmanente a la conciencia, se puede aclarar el sentido que aquel tiene para esta. Toda vivencia consciente puede entenderse, desde la perspectiva noemática de su contenido como "sentido", pero desde la perspectiva "activa" noética, se entiende como una "donación de sentido". La razón gesta, gracias a esa donación de sentido, una identidad y unidad en la corriente de vivencias. Incluso cuando el Husserl tardío vuelva sobre el "mundo de la vida" como fundamento del sentido, se trata de un mundo que carece de toda significación sin la labor de idealización de la conciencia. Por eso la fuente última de representaciones de conocimiento, de la reflexión del cognoscente, es el yo. Este yo es trascendental. El término es de procedencia kantiana y su sinónimo es "condición de posibilidad". O sea, el sujeto analizado no es un sujeto empírico concreto, sino que la fenomenología saca a la luz al sujeto trascendental que constituye aquello denominado mundo, que es la base de la correlación a priori de mundo y conciencia, o sea, del "objeto" y sus modos de darse a la conciencia.

Ahora bien, si el sujeto es esencialmente la apercepción de esta correlación intencional, ¿qué sucede cuando en su conciencia se da un fenómeno que muestra características análogas a las que él halla al percibirse a sí mismo? ¿Qué sucede cuando el fenómeno ante él no tiene las características de un objeto corporal (Körper) sino la de un cuerpo vivido (Leib)? Husserl afirma que el análisis de la intencionalidad desplegada en la conciencia de un alter ego debe, para ser fiel a sí mismo, permanecer en la inmanencia, pero al mismo tiempo reconocer la particularidad de ese fenómeno. La reducción del mundo trascendental a la esfera propia, monádica, no desconoce su direccionalidad, su tender a lo ajeno, su otro. Pero eso otro siempre es reconocido bajo el modo de una reducción a la conciencia misma. Cuando eso ajeno es otro yo, que por su sentido constituido por mí remite al sujeto mismo, pero por su modo de darse muestra un "análogo" a la experiencia propia del sujeto, entonces ese otro-yo muestra que despliega una vivencia intencional, por ejemplo respecto de su cuerpo vivo, análoga a la propia (Husserl, 1996:157). El dominio de sí del otro, los predicados que de él surgen, etc., son vistos siempre a partir del yo. En ese sentido, el sujeto sigue siendo constituyente del otro, aunque efectivamente esa alteridad se muestre ajena (Husserl, 1996:177). Los yoes pueden constituir una comunidad, que será siempre comunidad de mónadas que permanecen en su encierro. El otro no carece de originalidad en su manifestación, pero debido a la esencia intencional de la conciencia, su mundo propio será siempre sólo representado por el sujeto, mónada originariamente constituyente (Husserl, 1996:196).

\subsection{Existir como cura (¿sui?) en Heidegger}

El punto de partida de Heidegger es la refutación del intelectualismo de su maestro y la comprensión del modo de ser humano como una praxis vital relacional. Heidegger acaba con la comprensión moderna del sujeto realizada desde una conciencia trascendental, objetivante y donadora de sentido, y entiende fenomenológicamente - que aquello de lo que la "conciencia" es conciencia-de es el ser, y eso significa que el ser humano no es una "interioridad" constituyente, sino un lugar donde, a partir de su praxis y comprensión, el ser se manifiesta. Si 
cada ente, cada cosa que está siendo, tiene un modo de relacionarse con el ser, el modo de ese ente llamado humano es la apertura a la comprensión del ser. Aunque el interés de su obra principal Ser y tiempo no es antropológico sino ontológico, Heidegger conduce ese análisis de la manifestación del ser desde el "lugar" de la manifestación, el existir del ser humano, Dasein. Este término es un indicio más del intento heideggeriano de ruptura con lo que luego llamaría "historia de la metafísica", y que se caracterizaría por el olvido de la verbalidad del ser y de la diferencia ontológica de ser y ente, su reducción al mundo de las cosas y la búsqueda de algún fundamentum inconcussum subyacente, efectuada por términos como "sustancia", "concepto", "sujeto" y "conciencia" (cf. Heidegger, 1990: 131,143).

Si el sujeto moderno constituía al objeto, representándolo, legalizándolo, donándole sentido y finalmente manipulándolo, el Dasein es un estado de apertura y comprensión del ser. Para exponerlo no se puede recurrir al modelo clásico de "categorías" (originalmente "acusar" y luego "esquema de proposición" capaz de reunir multiplicidades bajo conceptos generales), ya que estas no están a la altura de lo que se muestra en la existencia humana. Por eso usa "existenciales" (en otra traducción "existenciarios") como manera de exponer las estructuras del modo de ser del ser humano: disposición afectiva, comprensión, lenguaje, caída, cuidado, temporalidad, historicidad.

El Dasein existe, se relaciona con el ser, ante todo comprendiéndolo. La base de esa comprensión es la constitución fundamental del Dasein: su "estar-en-elmundo" (Heidegger, 2003:79). El Dasein es un ente, algo que está siendo, pero con la particularidad de que aquello de que se trata en ese estar siendo es la comprensión y realización de su ser en el mundo - desde siempre así manifestado y no por él constituido. Se trata de una experiencia de integralidad e integración, ya que el mundo le constituye al Dasein y no es un objeto constituido por su conciencia. Sería un error comprender ese "estar-en" fundamental como "dentro" o "al lado de". El Dasein no es un ente que entra en relación con otros, sino que ese modo de ser relacional es precisamente el del Dasein. De modo tal que el Dasein siempre conoce ya de alguna manera al "mundo", está en una precomprensión respecto de él, el mundo se da primariamente como aperturidad.

Pero hay diversos modos de relacionarse. En ese mundo en que está el Dasein, aparecen otros que muestran que su "sustancia" es también su existir mismo, cuyo "yo" también ha de ser interpretado existencialmente, o sea, como apropiación de posibilidades y despliegue de un proyecto, como realización de su temporalidad. Se trata de la experiencia de "coexistencia de los otros" (Mitdasein) (Heidegger, 2003:142). Ser en el mundo significa ver lo que a uno lo rodea en su contexto de aplicación, de modo tal que la comprensión primaria del mundo es práxica antes que conceptual: las cosas "están a la mano". Pero aparece un tipo de ente que tiene el modo de ser de Dasein: "es tal como el mismo Dasein que lo deja en libertad - también existe y existe con él" (Heidegger 2003:143). Ese "co"estar no significa una disposición locativa sino un modo común de ser: "estar en el mundo ocupándose circunspectivamente de él".

El Dasein del otro y el propio tienen en común su modo de ser: ocuparse del mundo. Cada uno es único en ello, irreemplazable, como lo demuestra la posibilidad más propia del ser humano: morir. Diversos seres humanos pueden 
co-estar, pero su modo más propio de hacerlo es dejándose mutuamente en libertad. Heidegger le reconoce al otro un modo diverso de atención, que no es ocuparse de sino solicitud por (Fürsorge), caracterizada por el respeto y la indulgencia. Lo que une a los diversos Dasein es su estructura común del "ser por mor del cual el Dasein mismo es como es" (Heidegger 2003:148). Hay una diferencia esencial entre la relación con otros y la relación con las cosas. Pero no se trata de "empatía", sino de la estructura común que - propia o impropiamente - todo Dasein realiza en su temporalidad: todo Dasein intenta ser "uno mismo", y si lo hace de modo propio significará asumirse a sí mismo expresamente. En eso consiste la libertad, en la posibilidad de decidir en vistas de la propia posibilidad.

¿Cómo es el Dasein de modo propio? O preguntándolo teniendo en cuenta que lo que le va en su ser al Dasein es precisamente su propio ser, ¿cómo se despliega la existencia en vistas del propio ser? ¿Cuál es el fenómeno unitario que atraviesa todo Dasein en su relación ya práxica y comprensiva del mundo? Habida cuenta de la experiencia de finitud que caracteriza todo existir, habida cuenta de la angustia que la misma genera en todo Dasein que piense su propio proyecto en vistas a su fin, Heidegger muestra que el Dasein "ya desde antiguo, hablando de sí mismo, se interpretó como cuidado (cura)" (Heidegger, 2003:205).

Si el Dasein es ante todo en la aperturidad del mundo, su primera actividad es el des-ocultamiento de los demás entes. El Dasein se-encuentra en la facticidad de su ser como arrojado al mundo, y compendiéndolo puede anticiparse en sus posibilidades. En vistas a las posibilidades en cada caso propias (Jemeinigkeit), no respecto de otros entes que no son él sino en vistas a su poder-ser, "le va" su ser. El ser no es su otro, sino lo que le permite su más propia identidad al Dasein. La fórmula de la estructura del cuidado es "anticiparsea-sí-estando-ya-en-medio-de" (Heidegger, 2003:214). Esta estructura no debe confundirse con la ocupación con los entes, ni con la solicitud por otros Dasein. Tampoco es una cura sui en sentido estoico, referida ya a un comportamiento concreto respecto de sí. Es en cambio la anticipación libre de poder-ser las propias posibilidades. Es en vistas a ese poderser que se estructura el cuidado también como realización de la temporalidad. El cuidado no está determinado por un ente o un acto concreto, sino que "antecede" ontológicamente a toda decisión concreta. Por supuesto que ese despliegue del cuidado sólo sucede "en tanto el Dasein que así se proyecta responde por sí mismo" (von Herrmann, 2004:207). Pero su responder no está predeterminado por ningún ente del mundo sino por la fidelidad a su existir, a la relación auténtica con su ser. Por eso Heidegger llama al modo auténtico y cuidante de ser del Dasein existencia. Y su realización cuidadosa implica comprenderse anticipatoriamente desde donde se está en vistas de la realización del ser. Sólo sobre la base del cuidado por el ser-propio es que puede entenderse toda relación lograda o no con las cosas y los demás. Pero esas relaciones no son ontológicamente primordiales, sino la relación con el ser.

En Ser y tiempo la característica esencial del Dasein es su estado de apertura al ser, y ese estado se manifiesta primariamente en su estructura característica de cuidado, por el cual desde su estar ya en-medio del mundo se anticipa. Ese proyectarse hizo que algunos intérpretes vean aún en Heidegger cierto subjetivismo, particularmente en contraposición con los textos posteriores a 1935, donde el 
ser humano es mucho más pasivo frente a la manifestación histórica del ser (cf. Löwith, 1956). Sin embargo, el Dasein es ek-stático, o sea, libre de toda noción sustancial o subjetivista. Está "fuera de sí", en el sentido de pararse y realizarse a la luz del ser. Esa es la "trascendencia" que el análisis heideggeriano propone, y desde cuya crítica nace el pensamiento de Levinas, pues éste no entiende que existir a la luz del ser sea una verdadera trascendencia sino un proyectarse aún ligado a la ontología de la inmanencia característica de occidente.

\section{Una brecha en el orden del ser: la subjetividad levinasiana}

Levinas encuentra en Heidegger un "clima" opresivo: la constante presencia de sí a sí, la imposibilidad de evadirse del conatus essendi, del impulso a ser propio. La experiencia de ser-ineludible se expone con la metáfora del insomnio en la noche cerrada: sin límites, puro existir. Hay una dificultad en la interpretación de Levinas (genitivum subjectivum) pues éste entiende al ser al mismo tiempo como aquello diverso a y presente en todo ente, un fluir previo y anónimo, y como aquello que el sujeto querría mantener a toda costa como suyo propio (esta tensión puede verse en Levinas, 1993:83). Por eso ve al mismo tiempo en Heidegger una filosofía de la neutralidad impersonal y el mismo impulso base de la violencia característica de la filosofía y las instituciones occidentales. Pero también hay una dificultad en la interpretación de Levinas (genitivum objectivum) que le lee comprendiendo todo modo de ser como malo, como si Levinas estuviese negando, al modo de Schopenhauer, toda bondad a ser. De hecho, la sensibilidad de Levinas - proveniente de una experiencia-otra: su judaísmo - le permite no sólo criticar la posibilidad de anonimato y violencia inherente al ser de la filosofía occidental
- y a toda ciencia e institución -, sino también identificar los momentos en que una alteridad absoluta se mostró al pensamiento totalizador poniéndolo en cuestión: el Bien que Platón pone más allá de las esencias, el Infinito que Descartes ubica en la intimidad del sujeto.

Su método también es fenomenológico, pero la fidelidad a esa exposición de aquello que se manifiesta le lleva más allá de la manifestación misma. Atender a la manifestación del Otro significa "trastornar" el punto de partida de Husserl, o sea, que toda conciencia es conciencia de algo a lo que se le dona sentido. El Otro significa "un bouleversement de l'intentionalité, un retournement, une conversion (Levinas, 2005:279, 272, 277), une inversion, une subversión" (Levinas, 1987:235,241; cf. Casper, 2008:48). Atender al Otro significa también entender que la subjetividad no nace como apertura al ser anónimo sino como respuesta a Otro concreto, nominado y nominante, por cuya de-nominación y re-clamo el sujeto recibe como un don su identidad, como un Nombre.

La pregunta de Levinas no es sólo cómo surge "lo mismo", la identidad, en el orden del ser, sino cómo se manifiesta, aún en el seno de un intento totalizante y reductivo, el infinito, o sea la alteridad insubordinable a algún concepto del Otro. El método, a diferencia de la modernidad, no implica tomar un punto de partida indudable desde el cual reconstruir un conocimiento. En cambio vuelve sobre el "fenómeno" estudiado desde diversas perspectivas, "poniendo en escena" lo que el fenómeno "dice", incluso aquello que escapa al sujeto y su donación de sentido, y desfasa al propio fenómeno. El sujeto no es ya constituyente de mundo sino constituido por otro, a-sujetado. Si el hilo conductor de estas páginas es el surgimiento de la subjetividad y 
su relación con el Otro, un recorrido posible por la obra de Levinas podría estructurarse con tres pasos. En primer lugar, la manifestación de la exterioridad separada a la sensibilidad del sujeto; en un segundo momento la estructura responsorial de la subjetividad humana; y finalmente el orden de la temporalidad que la relación con el Otro deja traslucir.

\subsection{Separación y sensibilidad}

El problema de la "evasión" del ser neutral y anónimo es retomado en la posguerra por Levinas, particularmente en las conferencias publicadas como El tiempo y el Otro. Allí comienza un análisis que concluye en Totalidad e Infinito, donde muestra la insuficiencia de la comprensión de la subjetividad humana como hipóstasis. Con ese primer término intenta mostrar, en contraposición con Heidegger, la posibilidad de separación y no meramente de distinción de un existente en medio de la existencia (Levinas, 1993:83). ¿Cómo se asienta en medio del continuo del existir - "hay" - un existente? Los primeros análisis le llevan a una suerte de "apropiación" que sucede en la hipóstasis: la libertad de comenzar, de obrar (Levinas, 1993:91). El comienzo del actuar se hace ante todo por la condición de menesterosidad del sujeto, su "vivir-de" (Levinas, 1995:129). Al obrar, al comenzar una actividad de satisfacción de necesidades, el sujeto genera un ámbito propio, una morada, donde vive su independencia y su gozo: una primera experiencia de separación. El gozo proviene de la necesidad satisfecha. El sujeto re-presenta objetos, les manipula para sus necesidades, y en ese trabajo se reconoce a sí como un sí Mismo. La sensibilidad como pasividad, en este caso de una necesidad inherente, le permite al sujeto relacionarse con los elementos antes de toda representación. La dependencia primera muestra, al mismo tiempo, la pasividad del sujeto y su actividad posterior, por la cual se establece su hipóstasis: separación y gozo.

Sin embargo, en ese trabajo de separación y gozo permitido por la sensibilidad, aparece también una alteridad no representable, no tematizable. A diferencia de la necesidad que indica siempre un objeto de satisfacción finito, la alteridad genuina se muestra como "objeto" de deseo, pero de un deseo insaciable, infinito. Se trata del Otro que se manifiesta como rostro. Ese contacto muestra una pasividad "más pasiva que toda pasividad antitética a un acto", previa a y fundante de toda representación, libertad y actividad. Por eso para Levinas (1995:198,214) la relación con Otro es esencialmente ética y demanda una respuesta capaz de permitir su vida. No es una relación de tematización o donación de sentido de una conciencia trascendental, pues se muestra a modo de un llamado, una expresión que afecta a la propia sensibilidad de modo tal que ésta no puede volver sobre sí con su acción, y que por ende escapa toda representación o posesión. Muestra precisamente que el supuesto aislamiento gozoso con que se describía la subjetividad es, si no falso, al menos insuficiente y ciertamente no fundamental. Si la separación lograda por el gozo indicaba cierta soberanía del sujeto gozoso, la separación inaugurada por la relación con el Otro, que entra en relación con el sujeto llamándole antes de toda decisión propia, es una relación con el ab-soluto.

Lo absoluto del cara-a-cara (Levinas, 1995:199) indica su infinitud, o sea, su irreductibilidad a algún sistema totalizante - conceptual u otro. No se trata de una alteridad accidental, sino de una separación real. Y esa expresión de un rostro sólo es respondida por la 
responsabilidad inmediata. Cuando el Otro aparece cara-a-cara y se muestra rompiendo toda capacidad propia de donación de sentido, rompiendo todo continuum en el ser, el sujeto reconoce un "tránsito", una distancia manifestada en "el discurso, la bondad, el deseo" (Levinas, 1995:63), que ninguna actividad humana puede dar cuenta acabadamente. Por eso toda obra está condenada al fracaso en tanto es insuficiente, pues el deseo por ese Otro que se manifiesta y al que se quiere dar respuesta, no puede satisfacerse al modo de una necesidad cuya satisfacción produce gozo. Es en cambio una relación asimétrica con la infinitud de un ser separado.

Desde estos análisis primeros de la relación con el Otro en su alteridad irreductible se pueden abrir dos líneas de trabajo. En primer lugar, y muy acorde con la herencia fenomenológica, el trabajo de Levinas en Totalidad e Infinito sigue siendo el análisis de lo que sucede a la conciencia, en este caso ante el Otro: el "pensamiento" y la "interioridad" no son representación del Otro sino su "producción", o sea, una relación que se conoce sólo en tanto es efectuada. "La alteridad sólo es posible a partir del Yo" (Levinas, 1995:63,228), pues en el yo que se ha constituido el Otro manifiesta su llamado constituyente. Muy pronto Levinas reconoce los límites, las ataduras de este planteo. No lo rechaza, pero reconoce en él la "clausura" de un pensamiento que pretende desgarrar la filosofía tradicional con sus propias herramientas. Por ello radicalizará su análisis, planteando que no sólo consiste la subjetividad en una respuesta, instada por el deseo del Otro que el sujeto reconoce cuando este Otro le invoca, sino que la estructura misma del sujeto, previa a toda opción subjetiva, es la de ser-para-el-otro. Esta transitividad arroja también luz sobre los análisis levinasianos del tiempo. El tiempo es la relación con el Otro que no se acota a la propia finitud. El fracaso de la obra no implica su reducción a los límites de la propia existencia, al ser-para-la-muerte heideggeriano. Esa noción ya adquirida en El tiempo y el Otro y radicalizada con el análisis de eros y de la fecundidad en Totalidad e Infinito, alcanzan su mayor expresión en De otro modo que ser con la noción de diacronía.

\subsection{Ser-para-el-Otro}

De diversos modos la filosofía ha intentado mostrar el carácter de "propiedad" de la existencia propia. Quizás los términos más conocidos sean aquellos heredados de Hegel y tomados por Sartre, donde se propone, para la plena apropiación de sí en las propias posibilidades, el paso del "en sí" al "para sí". Heidegger, con una filosofía no subjetivista, lo plantea como "el carácter de ser-cada-vez-mío [Jemeinigkeit]" (Heidegger, 2003:68), o sea, como una relación con el ser como posibilidad más propia. Levinas, en cambio, des-apropia la subjetividad. La identidad de ésta, su unicidad irrevocable, no está en asumir las propias posibilidades, sino en responder al llamado del Otro. Pero esa respuesta no es unívoca.

El primer modo de entender esa relación responsorial con el Otro es mediante el deseo. El deseo es la apertura al otro más fundamental de la sensibilidad. Su menesterosidad característica, su necesidad, no es su estructura más originaria, sino su deseo. El primer modo con que Levinas lo expresa es la experiencia erótica, el deseo de lo deseable. Ese deseo hace de la identidad un círculo que no se cierra, una noidentidad que vive, respira, gracias a la elección de responsabilidad por el Otro. A esa elección se da respuesta, pero todo "Dicho", palabra o logos, 
toda construcción racional, es siempre imprescindible y limitada. En lo Dicho late un Decir, un intento siempre frustrado de co-responder al llamado, a la elección del Otro.

El individuo es elegido pasivamente, es rehén (Levinas, 1987:180; Casper, 2008:22). Es rehén porque "la subjetividad en tanto que lo otro dentro de lo mismo, en tanto que inspiración, es la puesta en entredicho de toda afirmación 'para sí', de todo egoísmo que renace dentro de la propia recurrencia" (Levinas, 1987:179). O sea, la subjetividad no puede volver a sí y afirmarse para sí, al modo en que Hegel piensa toda la historia del Espíritu, no porque no quiera sino porque no puede. Porque su unicidad significa tomar un lugar que sólo esa subjetividad puede tomar: la respuesta que sólo ella puede dar al otro que la inhabita. El sujeto es constitutivamente rehén de ese Otro.

Ese Otro, que aparece primero como exterioridad deseable, desborda esa primera manifestación al revelarse la condición de rehén que caracteriza a la subjetividad. Si en Totalidad en Infinito se resalta el régimen de separación del Otro, en De otro modo que ser el Otro se ve en la "proximidad": el prójimo, empero, no es el cercano - en el sentido de no-lejano -, sino el que con su llamado está presente en el sujeto como su identidad más profunda, más íntimo a mí que yo mismo. En Totalidad e Infinito la responsabilidad, respuesta al rostro infinito que manda no matar o dar vida, es relación con una exterioridad, con el Otro "absuelto", absolutamente separado de uno mismo. El mismo movimiento conduce en De otro modo que ser al llamado del Otro como una huella, ausente presencia del Otro en el sujeto.

Se trata de una "asignación" que el sujeto porta en sí al modo de una herencia
- a posteriori admisible o rechazable, pero recibida pasivamente, sin actividad propia -, y que es iniciada por una "proximidad no erótica". Se trata de un "deseo de lo no-deseable, de un deseo del extraño en el prójimo fuera de la concupiscencia" (Levinas, 1987: 194). Por eso el Otro obsesiona con su llamado. Si sólo fuera deseo de lo deseable permanecería latente el riesgo de una justificación egoísta de la respuesta. Quizás sea ese el sentido más profundo del utilitarismo presente en las éticas neo-racionalistas. En cambio, el Otro que reclama es extraño, extranjero, indeseable. Llama y la respuesta al llamado no "satisface" al sujeto. Es un traumatismo. Sin intencionalidad "terapéutica", pero con profundas consecuencias en ese ámbito, Levinas anota "el alma es el otro en mí. El psiquismo, el uno-para-el-otro puede ser posesión y psicosis; el alma es ya simiente de locura" (Levinas, 1987:126).

La "unicidad" del sujeto es su respuesta a la alteridad que respira en su interior. El sujeto está vocado por ella, y al responder es-para-ella. Todo Decir, que en lo Dicho late, intenta responder al otro. Pero responder al Otro es responder por el Otro. Ser su garante. No es una mera actitud o un compromiso (Levinas, 1987:210). Eso significaría devolver la relación a las capacidades del sujeto. Ser garante del Otro, su rehén, significa estar expuesto sin retorno. En última instancia, ser rehén del Otro significa darse por él, substituirse por él (Levinas, 1987:163). No hay que entender las palabras de Levinas ni como un misticismo ni como algún tipo de tendencia estoica al sacrificio, pues ambos son explícitamente rechazados. Es en cambio algo que, al igual que el tiempo, pasa al sujeto "a pesar suyo". "En mí ... se abre la comunicación" (Levinas, 1987:199) no porque el yo analice cómo se comunica con Otro, sino porque el yo descubre que no sería si el Otro no estuviera ya en él. 
Esa sujeción significa ser-para-el-Otro, una transitividad por la que el yo recibe su identidad heterónomamente. La libertad, la autonomía, la conciencia, y también las instituciones y las jurisprudencias, son todas ellas actividades necesarias pero "ontológicamente" posteriores. Lo primero es el Otro y su llamado electivo, y la respuesta en que ese llamado se pone en escena es je suis á toi.

Pero ¿cuándo recibió el sujeto ese llamado?, ¿cuándo se dio ese encuentro?

\subsection{El tiempo del Otro}

Los análisis del tiempo de Husserl y Heidegger son congruentes con sus fenomenologías. Husserl entiende al tiempo como conciencia inmanente del tiempo, de las vivencias ligadas en un flujo de donaciones a la conciencia. El tiempo original no es cronométrico, sino que la métrica, la medición de la duración, presupone ya el despliegue de la conciencia que percibe, retiene y protiende en un continuum. Por su parte, Heidegger utiliza en Ser y tiempo el término temporalidad como horizonte de comprensión del ser y despliegue de la existencia. La estructura del cuidado es la estructura misma de la temporalidad del Dasein: anticiparse-siendo ya-en medio de. Aunque los tres tienen igual fundamentalidad, el tiempo genuino reconoce según Heidegger un primado al futuro: anticiparse en vistas a la muerte. Así la temporalidad se anticipa a la muerte como posibilidad más propia, como posibilidad de comprensión del serpropio (futuro), en la repetición se vuelve a las posibilidades del existir ya sido, y en el presente se ubica en la apertura de posibilidades actuales.

Heidegger le provee a Levinas de un método de desformalización del tiempo, pues a diferencia de Kant, el tiempo no es una forma pura de la intuición, una estructura formal vacía por la cual la conciencia organiza lo que aparece en una sucesión, sino una experiencia desde la facticidad del Dasein como su modo de temporalización, como éxtasis-hacia sus posibilidades propias - que serán, son, y fueron (y en eso consiste el Dasein). Pero mientras para Heidegger el temporalizarse genuino es en-vistas-a la posibilidad de ser más propia de la muerte, propiedad que en cada caso es mía propia, ya muy temprano ve Levinas que la muerte "pasa", que no se asume (Levinas, 1993:114). Ante ella se pierde dominio, es un advenimiento. La relación con la muerte, con el porvenir, advierte Levinas, tiene la misma estructura que la relación con la alteridad. "La relación con el porvenir es la relación misma con otro. Hablar de tiempo a partir de un sujeto solo, de una duración puramente personal, es algo que nos parece imposible" (Levinas, 1993:117).

Pero no se trata de que nuestra relación con el tiempo, nuestro modo de temporalizarnos, tenga una estructura "análoga" a la de nuestra relación con el Otro. En cambio, en Totalidad e Infinito el tiempo será la relación misma con el Otro. El Otro es infinito, y por ende nuestra relación con él (con su demanda) es una infinición (Levinas, 1995:288). Pero para que haya tiempo debe haber algún origen, un punto en medio del continuum anónimo del ser. Esa es la relación con el Otro. No es la relación con la propia muerte lo que abre la temporalidad genuina, sino con el Otro que a la vez es infinito en su inconceptuabilidad pero finito en su mortalidad. La relación con ese Otro, recibirlo, comienza una temporalización que "va más allá de lo posible" (Levinas, 1995:288). El yo que se relaciona con esa posibilidad más allá de sus posibilidades, más allá de su muerte, descubre una "discontinuidad" (Levinas, 1995:289). 
La discontinuidad es ante todo la posibilidad de la novedad, la posibilidad de comenzar, de que advenga el novum, pensado sobre todo como tiempo sin mal. Levinas no lo sabe, pero otras tradiciones también buscaban la "tierra sin mal" (Galimberti, 2003). Levinas ve en la fecundidad una señal de ese ser para más allá de la muerte - los hijos son "el futuro sin mí". Pero la temporalidad es sobre todo "paciencia", "envejecimiento" como modo de responder al Otro a la espera de la plenitud imposible de la relación. El tiempo puede exponerse formalmente como secuencia continua de muerte y resurrección de instantes, "pero tal estructura formal supone la relación del yo con el Otro" (Levinas, 1995:291).

Si en Totalidad e Infinito la discontinuidad se piensa primariamente en vistas al futuro, De otro modo que ser profundiza sobre el origen an-árquico de esa paciencia y envejecer. El tiempo indica una pasividad subjetiva. Pasividad porque el sujeto no puede recuperar con un acto suyo al "arjé", al origen de esa relación con el Otro. Es "inmemorial", no porque la memoria sea débil, sino porque el encuentro con Otro, la discontinuidad que origina el tiempo, es diacrónica (Levinas, 1987:88). Nuevamente se hace patente la herencia judía con temas como creación y futuro mesiánico, o sea, "tiempos" en función de los cuales el sujeto se temporaliza pero de los cuales no fue ni será coetáneo. La respuesta que constituye la subjetividad no es el cumplimiento de una cláusula contractual, tratado en cuya firma el sujeto y el Otro estuvieron presentes. El Otro que se manifiesta próximo traumatiza al sujeto con una investidura, una responsabilidad heterónoma, un llamado no fijable cronométricamente, pero por cuya respuesta el llamado deviene alguien (Levinas, 1987:107). Se trata de la "elección" del Otro, que está ya en el sujeto de modo inmemorial - Otro-en-el-mismo -, pero que llama a una realización de la subjetividad como respuesta (Levinas, 1987:71). Pero aquello a lo que se responde no es una "presencia": ni el Otro es reductible a la presencia ni se muestra en la presencia de sí a sí en la conciencia. Se trata, en cambio, de una "huella", "intriga", imposible de reunir bajo el orden de lo sincrónico, del "tiempo común" (Levinas, 1987:128).

La temporalidad, según Levinas, no se da en vistas al ser, cuya esencia es "temporalización del tiempo, diastasis de lo idéntico y su recuperación o reminiscencia, unidad de apercepción" (Levinas, 1987:77). El sujeto no se temporaliza en vistas de una reunión en la presencia, sino como paciencia, padecimiento de una respuesta por la elección que Otro hizo del sujeto. Elección que se muestra diacrónica, inmemorial, pero cuya respuesta ha de darse en - y como - el despliegue de la temporalidad.

Naturalmente la filosofía levinasiana del Otro abre muchas preguntas. ¿Si no es en términos "occidentales", "filosóficos", con qué términos ha de abrirse esta experiencia? ¿Puede hablarse de "experiencia" si ningún concepto la nombra? Más aún, ¿es el existir propio necesariamente un conatus violento? ¿Es pensar el ser siempre una reducción de la experiencia de la alteridad? Por ejemplo, el Heidegger posterior a 1935 entenderá que no ha de abrirse el sentido de ser desde la estructura del Dasein sino desde su manifestación y donación histórica. Hay (es gibt) ser, y esa exhuberancia de donación no debe confundirse con el término "il y a" en Levinas, donde se alude al anonimato del puro existir sin existente, sin un sujeto que se haya separado del continuum del ser, identificándose como sujeto, y haciéndolo precisamente a partir de Otro. 
Estas preguntas a Levinas son hechas también desde nosotros. Ciertamente Levinas ya permite reconocer la alteridad desde siempre latente en el pensamiento occidental, por ejemplo dando lugar a nociones provenientes de su tradición judía. Pero en términos de Levinas apropiados por filosofías latinoamericanas, esta reconstrucción ha sido hecha desde "lo mismo", o sea, desde la tradición del pensamiento europeo, en el cual seguimos "clausurados", o sea, tratando de ir más allá de sus límites pero imposibilitados por un lenguaje que se nos resiste. ¿Qué lenguaje cabe a la alteridad? ¿Cómo poner en escena que el Otro entra ya no como un elemento exótico sino constitutivo de la identidad, como lo fuera el caso del judaísmo en occidente y como deben serlo, en un trabajo de reconocimiento aún inconcluso, las diversas tradiciones que confluyen en lo que hoy se denomina Latinoamérica? Se abre aquí una serie de interrogantes en torno a la pregunta de qué tipo de "racionalidad" la alteridad permite y su relación con la "sensibilidad" (cf. Asselborn, 2010).

\section{REFERENCIAS}

Asselborn, Carlos (et al.) (2009) Liberación, estética y política. Aproximaciones filosóficas desde el Sur. Córdoba: EDUCC.

Casper, Bernhard (2008) Pensar de cara a otro. Elementos del pensamiento de Emmanuel Levinas. Córdoba: EDUCC.

Galimberti, Umberto (2003) La terra senza il male. Jung, dall'inconscio al simbolo. Milano: Feltrinelli.
Heidegger, Martin (1990) Identidad y diferencia. Barcelona: Anthropos - (2003) Ser y tiempo. Madrid: Trotta.

Hinkelammert, Franz (2005) "Prometeo, el discernimiento de los dioses y la ética del sujeto, en Utopía y praxis latinoamericana". En Revista Utopía y praxis latinoamericana, de la Universidad del Zulia, Maracaibo, Venezuela. Año 10, No 31, octubrediciembre, pp. 9-36.

Husserl, Edmund (1992a) Ideas relativas a una fenomenología pura y una filosofía fenomenológica. México: FCE.

_ (1992b) Grundprobleme der Phänomenologie. Hamburg: Meiner. - (1996) Meditaciones cartesianas.

México: FCE.

Levinas, Emmanuel (1987) De otro modo que ser, o más allá de la esencia. Salamanca: Sígueme.

w el tiempo y el otro.

Barcelona: Paidós.

(1994) Dios, la muerte y el tiempo. Madrid: Cátedra.

_. (1995) Totalidad e Infinito.

Salamanca: Sígueme.

- (2005) Descubriendo la existencia con Husserl y Heidegger. Madrid: Síntesis.

Löwith, Karl (1956) Heidegger, pensador de un tiempo indigente. Madrid: Rialp.

Rosenzweig, Franz (1997) La estrella de la redención. Salamanca: Sígueme.

Von Herrmann, Friedrich-Wilhelm (2004) Subjekt und Dasein. Frankfurt: Klostermann. 
